\title{
DAS WORDEN-PASSIV ALS SYNTAKTISCHE VERSTÄARUNG IM DEUTSCHEN
}

Zur Problematik des althochdeutschen und mittelhochdeutschen Passivs*

Auf der frühesten Entwicklungsstufe, welche durch schriftliche Überlieferung belegt werden kann, d.h. in der althochdeutschen Zeit (Ahd.), wurde das Passiv mit Hilfsverben "sein" (ahd. uuesan/wesan) oder "werden". (ahd. uuerdhan/werdan) und dem Partizip II gebildet. Als ältere Variante gilt die wesan-Periphrase; das Hilfsverbum werdan aber hat sich etwas später, jedoch noch in der vorliterarischen Periode, aus dem ursprünglichen Aspektsystem losgelöst, um sich im System der GENERA VERBI zu integrieren, und hat schon im Althochdeutschen als eines der Hilfsverben zur Bildung des Passivs Fuss gefasst. (Nach z. B. OEberg 1907, Valentin 1986).

Im Althochdeutschen (8. bis 12. Jh.) bildeten beide Konstruktionstypen des Passivs ein Paradigma, wobei anzunehmen ist, dass die semantische Dissimilation noch nicht eingetreten war. Es zeigte sich lediglich ein aspektueller Unterschied und gewisse temporale Abgrenzungen.

Das Passiv wies auf dieser Entwicklungsstufe noch einen stark ausgeprägten nominalen Charakter auf, d.h. Tempus und Aspekt wurden allein durch die Form des Hilfsverbums bestimmt, das Partizip II hingegen war lediglich eine nominale Ergänzung. Beispiel: wirdit arslagan bedeutet wörtlich er wird ein Erschlagener, nicht aber wird-sein. (So Behaghel, 1924-1932.) Auch die Entwicklung der Tempora hatte noch nicht die Stufe erreicht, welche einen unmittelbaren Vergleich mit der heutigen Konzeption zuliesse.

Einige Experten für das althochdeutsche Passiv befürworten neuerdings die Theorie, nach welcher der Hauptunterschied zwischen den Hilfsverben werdan und wesan daring lag, dass ersteres einen Vorgang als solchen ausdrückte und dadurch die Fähigkeit besass, in Verbindung mit dem Partizip II einen Vorgang auszudrücken, welcher in einem gewissen Augenblick in einen durch das Partizip II ausge-

* Der vorliegende Artikel is eine Zusammenfassung der Magisterarbeit der Autorin mit dem Titel "Das Passiv als syntaktische Erscheinung im Deutschen". Die Forschungsarbeit wurde an der Philosophischen Fakultät in Ljubljana unter Betreuung von Prof. Dr. Janez Orešnik als Teil des Magisterstudiums der Autorin erarbeitet. Die Verteidigung der Arbeit fand am 15. April 1988 statt. 


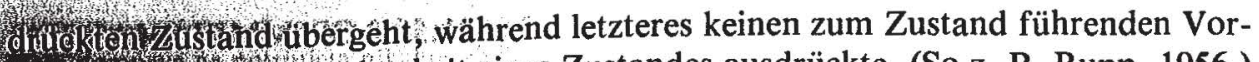
gang sondên die Gegebenheit eines Zustandes ausdrückte. (So z. B. Rupp, 1956.) 1.

1 101e wesan-Periphrase wies jedoch noch keinen Gebrauch in der Bedeutung von "St. Wworden auf, da dieser temporale Bezug sogar im Aktiv noch fehlte und sich erst in der mittelhochdeutschen Zeit behauptete.

Einer anderen Theorie zufolge sollten werdan und wesan noch keine richtigen Hilfsverben gewesen sein. Im gegenseitigen Verhältnis zeigten sie sich nicht als zwei aspektuelle Varianten desselben Verbs, nämlich werdan mit ingressiver und punktueller Bedeutung, hingegen wesan zur Bezeichnung eines durativen Vorganges. Für eine solche Konstellation besassen sie wahrscheinlich noch zuviel an lexikalischer Bedeutung. Sie waren auch noch nicht grammatikalisiert, da sie an bestimmten Stellen des Konjugationssystems in komplementärer Distribution, vor allem im Präteritum, an anderen Stellen dagegen in Opposition zueinander standen. (So Valentin 1986.)

Aus dem oben Gesagten is zu ersehen, dass das Verhältnis zwischen den beiden Konstruktionsytpen des Passivs im Althochdeutschen noch weitgehend ungeklärt ist. Erforderlich wären vor allem eingehendere Untersuchungen der Umstände, unter welchen die semantische Dissimilation und die Grammatikalisierung der beiden Hilfsverben erfolgten. Mit Sicherheit können wir jedoch schon jetzt feststellen, dass in der mittelhochdeutschen Zeit eine wesentliche Veränderung im Konjugationssystem des Passivs zu verzeichnen ist.

Entwicklung des worden-Passivs

Im Mittelhochdeutschen verzeichnen wir das Eintreten eines neuen Subsystems in das Passiv-Paradigma, welches bis heute weitgehend erhalten blieb. Es traten nämlich passivische Perfektformen auf, und zwar in Analogie zu früher entstandenen aktivischen Perfektformen. Analog mit der aktivischen Perfektform vom Typ er ist gekommen erhielt nun auch die Passivkonstruktion vom Typ er ist geschlagen perfektive Bedeutung. Das Hilfsverb werden behauptete sich mittlerweile im Präsens und Präteritum, parallel dazu entstanden aber in mittelhochdeutscher Zeit das Perfekt und das Plusquamperfekt mit ist, war worden. Ein solcher Zustand verweist auf die Neigung, werden in allen Passivformen durchzusetzen, allmählich kommt es zur Grammatikalisierung der mit werden und Partizip II gebildeten Periphrase, diese verfügt jetzt über ein Perfekt und verhält sich wie ein normales Verb (Dal, 1966; Valentin, 1986).

Die ältesten - allerdings noch relativ seltenen - Belege des worden-Passivs stammen aus dem 14. Jahrhundert. Vorerst traten in Konstruktionen mit worden Adjektive auf, erst etwas später das Partizip II. Beispiele: Darumb ist er wol drei 
stund ser geslagen und worden wund, Kaufringer III, S. 641-2; Ich bin nie von euch betrubt worden... ich bin all weg von euch gebezzert worden, Nonne von Engelthal, S. 7. (Beispiele angeführt nach OEberg, 1907.)

In der frühesten Entwicklungsperiode gab es eine Koexistenz der Konstruktionen mit ist + Partizip II und mit ist + Partizip II + worden, wobei beide Konstruktionstypen bedeutungsgleich waren. Beispiele: Da ist Anno 1115. ein kuenstliche, beruembte Brueck auffgerichtet worden, Faustbuch 1587, S. 230; die Haeuser sind all gemalt, und neben der Keyserlichen wonung eine hohe schul uffgericht, Faustbuch 1587, S. 231. (Beispiele angeführt nach OEberg, 1907.)

Bedeutungsgleich waren die beiden Konstruktionstypen jedoch nur in Fällen, in denen sie sich mit einem perfektiven Partizip II verbanden. In Fällen, wo das Partizip II imperfektiv war, gingen allmählich die Formen ohne worden ins Präsens über. Worden mit imperfektivem Partizip II drückt nämlich keine perfektivische Bedeutung im Sinne eines gegenwärtigen Resultates aus, sondern sogar eine deutliche $\mathrm{Ne}$ gation dieser Zeitreferenz. Der Druck des Systems führte schliesslich die Entwicklung der Passivmunschreibung mit worden zu einem Zustand, welcher dem heutigen sehr nahe steht: bei perfektiven Partizipien sind beide Konstruktionstypen (mit oder ohne worden) komplementär, dagegen ist worden bei imperfektiven Partizipien in perfektivischen Tempora (im Perfekt und Plusquamperfekt) obligatorisch. (So OEberg, 1907.) In solchen Fällen muss demnach zur Verdeutlichung die Konstruktion durch das Wort worden verstärkt werden. (Beispiel: er ist erschossen gegenüber er ist erschossen worden; es ist bewohnt gegenüber es ist bewohnt worden.)

Nach unserer Arbeitshypothese ist die Entwicklung der Passivumschreibungen für perfektivische Tempora als syntaktische Verstärkung zu werten. Diese Behauptung wird mit folgendem begründet:

Den schon bestehenden Passivumschreibungen in den perfektivischen Tempora wird das Wort worden hinzugefügt, wobei wir das Hinzufügen eines neuen Elementes als Verstärkung einstufen. Durch das Wort worden sind die perfektivischen Tempora des Passivs auch leichter dekodierbar.

Die periphrastische Konstruktion mit worden entsteht in zusammengesetzten Tempora, die ihrerseits kompliziertere grammatische Verhältnisse und dadurch eine günstigere Umwelt für verstärkte Konstruktionen darstellen, als das bei einfachen Verbaltempora der Fall ist.

Es wird angenommen, dass bei verstärkten Konstruktionen der Häufigkeitsgrad ihres Auftretens niedriger ist als bei entsprechenden nichtverstärkten Konstruktionen. Diese Annahme wird durch die relativ niedrige Häufigkeit des worden-Passivs in ältesten Quellen bestätigt. So konnte zum Beispiel OEberg im Trojanischen Krieg nur vier Belege für diese Konstruktion entdecken - die Dichtung um- 
fasst 49836 Verse. Ende des 14. und im 15. Jh. treten die Exemplare dieser Konstruktion in den Texten schon etwas häufiger auf. Im 16. Jh. war ihr Gebrauch schon üblich, wenn auch im Vergleich mit entsprechenden weniger verstärkten Konstruktionen immer noch selten. In Luthers Neuem Testament finden sich beispielsweise insgesamt nur sieben Beispiele.

Mit imperfektiven Partizipien II wird der Gebrauch des worden-Passivs obligatorisch, die weniger verstärkte Variante ohne worden zieht sich dagegen in eine "leichtere" grammatische Umgebung zurück - ins Präsens. Die Imperfektivität bewerten wir als eine "schwierigere" grammatische Umgebung, da sie einen grösseren Markiertheitsgrad aufweist als die Perfektivität.

Diachrone Vorhersagen bezüglich des deutschen worden-Passivs

Unserer Vorhersage zufolge sollten die Vollverben beim worden-Passiv im Durchschnitt "schwieriger" sein als die Vollverben bei anderen, weniger verstärkten Konstruktionen. "Schwierigkeit" bei Verben stellen vor allem Präfixe dar (ohne Präfigierung sind Verben "leichter"), dazu kommen noch Emphase, Affektwert und Intensität. "Schwierigere" Verben weisen in der Sprache eine niedrige Häufigkeit auf.

Da das worden-Passiv der Definition einer verstärkten Konstruktion entspricht, erwarten wir es häufiger im komplizierten Kontext.

Infinitive und die sogenannten absoluten Konstruktionen verhalten sich gegenüber Sätzen als geschwächte Konstruktionen, daher unsere Annahme, dass es in solchen grammatischen Umgebungen weniger worden-Passive geben wird. Den gleichen Zustand erwarten wir bei Modalverben, die ihrerseits auch leichtere grammatische Verhältnisse darstellen. Gegenüber Hauptsätzen verhalten sich Nebensätze als verstärkte Konstruktionen, daher werden dort mehr worden-Passive erwartet als in den Hauptsätzen.

Das Hinzufügen des präpositionalen Agens beim Passiv bedeutet Verstärkung, aus diesem Grunde erwarten wir beim worden-Passiv ein häufigeres Erscheinen des präpositionalen Agens als bei Passivumschreibungen ohne worden.

\section{Zusammenfassung}

Es ist anzunehmen, dass das worden-Passiv als verstärkte Konstruktion häufiger als das Passiv ohne worden mit präpositionalem Agens auftritt. Erwartet werden "schwierigere" Vollverben, weiters "schwierigere" grammatische Verhältnisse wie 
etwa vorwiegend Plusquamperfekt ${ }^{1}$, Plural, nichtdritte Verbalpersonen, Konjunktive, nichtbejahende Sätze und Nebensätze. (Aspektuelle Verhältnisse werden aufgrund des perfektiven Charakters der Konstruktion beiseite gelassen.)

\section{Die Einstufung des Korpus und Auswertungsmethode}

Die aufgestellten Vorhersagen hinsichtlich der Entstehung und Durchsetzung des deutschen worden-Passivs werden anhand eines sprachlichen Korpus überprüft. Die Wahl fiel auf Hans Jakob Christoffel von Grimmelshausens Simplicissimus. Unsere Entscheidung begründen wir mit der Tatsache, dass dieses Werk einen umfangreicheren zusammenhängenden und in sich abgeschlossenen Text aus dem 17. Jh. darstellt. Für unsere Textauswahl spricht auch der Umstand, dass es sich um keine Übersetzung, sondern um einen authentischen deutschen Text handelt, aus einer Zeit, in der das worden-Passiv sich schon dermassen entwickelt hatte, dass wir sein Paradigma schon als grammatikalisiert bezeichnen können.

Die Textanalyse basierte auf dem Vergleich zweier Samples, eines Grund- und eines Kontrollsamples. Ins Grundsample sind alle diejenigen Sätze aufgenommen worden, die Beispiele der untersuchten Konstruktion enthalten, während das Kontrollsample eine repräsentative Stichprobe aus den übrigen Sätzen des Korpus darstellt.

Jeder Satz des Grund- und Kontrollsamples wurde nach folgenden grammatischen Kriterien klassifiziert: Satzform und Satzart, Verbalperson, Numerus, Tempus und Modus. Bei Passivsätzen wurde zusätzlich noch das Vorhandensein bzw. Fehlen des präpositionalen Agens überprüft. Die Auswahl der berücksichtigten grammatischen Kategorien beruht auf den Eigenheiten des Grunsamples. Das Resultat einer solchen Satzanalyse waren Teilangaben über die Komplexität der grammatischen Verhältnisse, in welchen das worden-Passiv und andere im Vergleich schwächere Konstruktionen erscheinen. Nach allgemein gültigen statistischen Formeln wurde die Wahrscheinlichkeit errechnet, nach welcher beim worden-Passiv bzw. Passiv ohne worden und Aktiv einzelne grammatische Kategorien in ihrer komplizierteren (markierteren) Variante vorkommen. (Beispiel: Singular gegenüber Plural, Perfekt gegenüber Plusquamperfekt, Indikativ gegenüber Konjunktiv usw.)

Wenn die Vorkommenswahrscheinlichkeit des "markierteren" (komplizierteren) Wertes von einem grammatischen Parameter in einer verstärkten Konstruktion gegeben ist und wenn die Vorkommenswahrscheinlichkeit des "markierteren" (komplizierteren) Wertes von demselben grammatischen Parameter in der entsprechenden nichtverstärkten Konstruktion - oder generell ausserhalb der verstärkten Kon-

\footnotetext{
1 Bei der Kategorie des Verbaltempus vergleichen wir das Perfekt mit dem Plusquamperfekt, da die untersuchte Konstruktion in anderen Verbaltempora nicht vorkommt. Dabei fassen wir das Plusquamperfekt als schwierigere grammatische Umgebung auf als das Perfekt.
} 
struktion - gegeben ist, dann ist die erstgenannte Wahrscheinlichkeit grösser als die zweitgenannte Wahrscheinlichkeit und der Unterschied zwischen den beiden Wahrscheinlichkeiten ist statistisch signifikant.

Nach unserer Arbeitshypothese erwarteten wir eine grössere Vorkommenswahrscheinlichkeit komplizierterer Werte einzelner grammatischer Kategorien und im Durchschnitt schwierigere Vollverben beim worden-Passiv als bei sonstigen weniger verstärkten Konstruktionen. Auch hinsichtlich des präpositionalen Agens wurde angenommen, dass die Wahrscheinlichkeit seiner Anwesenheit beim worden-Passiv grösser sein würde als bei den übrigen Passiv-Konstruktionen.

Analyse einzelner worden-Passiv Belege aus dem Grundsample ${ }^{2}$

9/19 geboren wären worden

N: Pl.; SF: bejahend; P: dritte; T: Pqp.; M: Kon.; S: Nebensatz;

V: schwierig; ohne präp. S.

erzogen wären worden

N: Pl.; SF: bejahend; P: dritte; T: Pqp.; M: Kon.; S: Nebensatz;

V: schwierig; ohne präp. S.

$13 / 23$ ist nicht erzogen worden

N: Sg.; SF: nichtbejahend; P: dritte; T: Perf.; M: Ind.; S: Hauptsatz;

V: schwierig; mit präp. S.

16/6 zu recht gebracht worden waren

N: Pl.; SF: bejahend; P: dritte; T: Pqp.; M: Ind.; S: Nebensatz;

V: schwierig; mit präp. S.

$17 / 13$ verübet worden

N: Pl.; SF: bejahend; P: dritte; T: Perf.; M: Ind.; S: Nebensatz;

V: schwierig; ohne präp. S.

22/35 geholffen worden

N: Sg.; SF: bejahend; P: dritte; T: Perf.; M: Ind.; S: Nebensatz;

V: leicht; ohne präp. S.

2 Die erste Ziffer vor jedem Beispiel bedeutet die Seite, die zweite Ziffer dagegen die Zeile, in welcher sich das Wort worden befindet. Falls in einem Satz zwei oder mehrere Verben in der Passsivform mit worden auftreten, ist jedes getrennt analysiert, gemäss seiner Reihenfolge im Satz. Beispiele aus dem Kontrollsample sind nicht angeführt.

ABKÜRZUNGEN: N = Numerus; Sg. = Singular; Pl. = Plural; SF = Satzform; $\mathrm{P}=$ Person; $\mathrm{T}=$ Tempus; Perf. = Perfekt; Pqp. = Plusquamperfekt; $\mathbf{M}=$ Modus; Ind. = Indikativ; Kon. = Konjunktiv; $\mathrm{S}=\mathrm{Satz} ; \mathrm{V}=$ Verbum; präp. $\mathrm{S}$. = Präpositionalsubjekt 
39/1 gerathen worden wäre

N: Sg.; SF: bejahend; P: dritte; T: Pqp.; M: Kon.; S: Nebensatz; V: schwierig; ohne präp. S.

39/33 geplündert worden

N: Sg.; SF: bejahend; P: dritte; T: Pqp.; M: Ind.; S: Nebensatz; V: schwierig; ohne präp. S.

verbrennt worden

N: Sg.; SF: bejahend; P: dritte; T: Pqp.; M: Ind.; S: Nebensatz; V: schwierig; ohne präp. S.

$56 / 26$ geschrieben worden ist

N: Sg.; SF: bejahend; P: dritte; T: Perf.; M: Ind.; S: Nebensatz; V: leicht; mit präp. S.

$56 / 30$ abgeredt worden

N: Sg.; SF: bejahend; P: dritte; T: Pqp.; M: Ind.; S: Nebensatz; V: schwierig; ohne präp. S.

58/3 geplündert worden

N: Sg.; SF: bejahend; P: dritte; T: Perf.; M: Ind.; S: Nebensatz; V: schwierig; ohne präp. S

verbrennt worden

N: Sg.; SF: bejahend; P: dritte; T: Perf.; M: Ind.; S: Nebensatz; V: schwierig; ohne präp. S.

$61 / 9$ ist verleitet worden

N: Sg.; SF: bejahend; P: dritte; T: Perf.; M: Ind.; S: Hauptsatz; V: schwierig; mit präp. $S$.

$61 / 14$ verloren worden

N: Sg.; SF: bejahend; P: dritte; T: Pqp.; M: Ind.; S: Nebensatz; V: leicht; ohne präp. S.

63/6 aussgeplündert worden

N: Sg.; SF: bejahend; P: nichtdritte; T: Pqp.; M: Ind.; S: Nebensatz;

V: schwierig; ohne präp. S.

beschädiget worden

N: Sg., SF: bejahend; P: nichtdritte; T: Pqp.; M: Ind.; S: Nebensatz;

V: schwierig; ohne präp. S. 
$65 / 22$ ist gefangen worden

N: Sg.; SF: bejahend; P: dritte; T: Perf.; M: Ind.; S: Hauptsatz;

$\mathrm{V}$ : leicht; mit präp. S.

$65 / 30$ sey zertrennt worden

N: Sg.; SF: bejahend; P: dritte; T: Perf.; M: Kon.; S: Hauptsatz;

V: schwierig; mit präp. S.

sey verloren worden

N: Sg.; SF: bejahend; P: dritte; T: Perf.; M: Kon.; S: Hauptsatz;

V: leicht; mit präp. S.

66/11 erzogen worden

N: Sg.; SF: bejahend; P: nichtdritte; T: Perf.; M: Ind.; S: Nebensatz;

V: schwierig; ohne präp. S.

gewehnet worden

N: Sg.; SF: bejahend; P: nichtdritte; T: Perf.; M: Ind.; S: Nebensatz;

V: schwierig; ohne präp. S.

$68 / 13$ seye gegeben worden

N: Sg.; SF: bejahend; P: dritte; T: Perf.; M: Kon.; S: Hauptsatz;

V: leicht; ohne präp. S.

73/35 aufferzogen worden wäre

N: Sg.; SF: bejahend; P: dritte; T: Pqp.; M: Kon.; S: Nebensatz;

V: schwierig; ohne präp. S.

74/31 gefangen waren worden

N: Pl.; SF: bejahend; P: dritte; T: Pqp.; M: Ind.; S: Nebensatz;

V: leicht; mit präp. S.

75/7 wärst gehenckt worden

N: Sg.; SF: bejahend; P: nichtdritte; T: Pqp.; M: Kon; S: Nebensatz;

V: leicht; ohne präp. S.

$81 / 4$ gegeben worden wäre

N: Sg.; SF: bejahend; P: dritte; T: Pqp.; M: Kon.; S: Nebensatz;

V: leicht; ohne präp. S.

87/13 zerschlagen worden

N: Sg.; SF: bejahend; P: nichtdritte; T: Pqp.; M: Ind.; S: Nebensatz;

V: schwierig; ohne präp. S. 
88/35 agirt worden

N: Sg.; SF: bejahend; P: nichtdritte; T: Perf.; M: Ind.; S: Nebensatz;

V: schwierig; mit präp. S.

96/8 überredet worden

N: Sg.; SF: nichtbejahend; P: nichtdritte; T: Perf.; M: Kon.; S: Nebensatz;

V: schwierig; ohne präp. S.

97/18 nicht geladen worden

N: Sg.; SF: nichtbejahend; P: nichtdritte; T: Pqp.; M: Ind.; S: Nebensatz; V: leicht; ohne präp. S.

98/2 gesperret worden

N: Sg.; SF: bejahend; P: nichtdritte; T: Perf.; M: Ind.; S: Nebensatz;

V: schwierig; ohne präp. S.

100/9 wäre angestellt worden

N: Sg.; SF: bejahend; P: dritte; T: Pqp.; M: Kon.; S: Hauptsatz;

V: schwierig; ohne präp. S.

101/31 erlöst worden

N: Sg.; SF: bejahend; P: nichtdritte; T: Pqp.; M: Ind.; S: Nebensatz;

V: schwierig; ohne präp. S.

111/30 bin gehärtet worden

N: Sg.; SF: bejahend; P: nichtdritte; T: Perf.; M: Ind.; S: Hauptsatz;

V: schwierig; ohne präp. S.

112/1 gelassen worden wäre

N: Sg.; SF: bejahend; P: dritte; T: Pqp.; M: Kon.; S: Nebensatz;

V: leicht; ohne präp. S.

114/10 geboren worden seyest

N: Sg.; SF: bejahend; P: nichtdritte; T: Perf.; M: Kon.; S: Nebensatz;

V: schwierig; ohne präp. S.

$115 / 24$ vorgesprochen worden

N: Pl.; SF: bejahend; P: dritte; T: Pqp.; M: Ind.; S: Nebensatz;

V: schwierig; ohne präp. S.

116/38 dörfftestu verwandelt worden seyn

infinite Form: S: Hauptsatz; V: schwierig; ohne präp. S. 
119/2 geschnitzelt worden wären

N: Pl.; SF: bejahend; P: dritte; T: Pqp.; M: Kon.; S: Nebensatz;

V: schwierig; ohne präp. S.

120/5 erhoben worden

N: Pl.; SF: bejahend; P: dritte; T: Perf.; M: Ind.; S: Nebensatz;

V: schwierig; mit präp. S.

120/19 seyn begangen worden

N: Pl.; SF: nichtbejahend; P: dritte; T: Perf.; M: Kon.; S: Nebensatz;

V: schwierig; ohne präp. S.

seyn erfunden worden

N: Pl.; SF: nichtbejahend; P: dritte; T: Perf.; M: Kon.; S: Nebensatz;

V: schwierig; ohne präp. S.

$123 / 6$ ist gehalten worden

N: Sg.; SF: nichtbejahend; P: dritte; T: Perf.; M: Kon.; S: Hauptsatz;

V: leicht; ohne präp. S.

$123 / 21$ erobert worden ist

N: Sg.; SF: nichtbejahend; P: dritte; T: Perf.; M: Ind.; S: Nebensatz;

V: schwierig; ohne präp. S.

$z u$ wegen gebracht worden ist

N: Sg.; SF: nichtbejahend; P: dritte; T: Perf.; M: Ind.; S: Nebensatz;

V: schwierig; ohne präp. S.

131/37 zugelassen worden

N: Sg.; SF: bejahend; P: dritte; T: Perf.; M: Kon.; S: Nebensatz;

V: schwierig; ohne präp. S.

$132 / 2$ solte verhängt worden seyn

infinite Form: S: Nebensatz; V: schwierig; ohne präp. S.

solte zugelassen worden seyn

infinite Form: S: Nebensatz; V: schwierig; ohne präp. S.

132/6 erschaffen worden

N: Sg.; SF: bejahend; P: dritte; T: Pqp.; M: Ind.; S: Nebensatz;

V: schwierig; ohne präp. S.

132/11 erzogen worden

N: Sg.; SF: bejahend; P: dritte; T: Pqp; M: Ind.; S: Nebensatz;

V: schwierig; ohne präp. S. 
$132 / 30$ zu recht gebracht worden

N: Sg.; SF: bejahend; P: dritte; T: Perf.; M: Ind.; S: Nebensatz;

V: schwierig; ohne präp. S.

$133 / 12$ ist geholffen worden

N: Sg.; SF: bejahend; P: dritte; T: Perf.; M: Ind.; S: Hauptsatz;

V: leicht; ohne präp. S.

$141 / 11$ erfreut worden

N: Sg.; SF: bejahend; P: nichtdritte; T: Perf.; M: Ind.; S: Nebensatz;

V: schwierig; ohne präp. S.

$146 / 20$ vertrieben worden

N: Sg.; SF: bejahend; P: dritte; T: Pqp.; M: Ind.; S: Nebensatz;

V: schwierig; mit präp. S.

$147 / 3$ verbrennt worden

N: Sg.; SF: bejahend; P: dritte; T: Perf.; M: Ind.; S: Nebensatz;

$\mathrm{V}$ : schwierig; ohne präp. S.

149/33 ruinirt worden

N: Sg.; SF: bejahend; P: dritte; T: Pqp.; M: Ind.; S: Nebensatz;

V: schwierig; mit präp. S.

$154 / 11$ ist verbotten worden

N: Sg.; SF: bejahend; P: dritte; T: Perf.; M: Ind.; S: Hauptsatz;

V: schwierig; mit präp. S.

ist verwehret worden

N: Sg.; SF: bejahend; P: dritte; T: Perf.; M: Ind.; S: Hauptsatz;

V: schwierig; mit präp. S.

$158 / 25$ gemeldet worden

N: Sg.; SF: bejahend; P: dritte; T: Perf.; M: Ind.; S: Nebensatz;

V: schwierig; ohne präp. S.

164/20 erquickt worden

N: Sg.; SF: bejahend; P: dritte; T: Pqp.; M: Ind.; S: Hauptsatz;

V: schwierig; ohne präp. S.

$167 / 8$ angedeutet worden

N: Sg.; SF: bejahend; P: dritte; T: Pqp.; M: Ind.; S: Nebensatz;

V: schwierig; mit präp. S. 
167/24 wäre geboren worden

N: Sg.; SF: bejahend; P: nichtdritte; T: Pqp.; M: Kon.; S: Hauptsatz;

V: schwierig; mit präp. S.

wäre erzogen worden

N: Sg.; SF: bejahend; P: nichtdritte; T: Pqp.; M: Kon.; S: Hauptsatz;

V: schwierig; mit präp. S.

167/29 eingewieget worden

N: Sg.; SF: bejahend; P: dritte; T: Perf.; M: Ind.; S: Nebensatz;

V: schwierig; ohne präp. S.

$168 / 18$ errettet worden

N: Sg.; SF: bejahend; P: nichtdritte; T: Pqp.; M: Ind.; S: Nebensatz;

V: schwierig; ohne präp. S.

168/33 eingenommen worden

N: PI.; SF: bejahend; P: dritte; T: Pqp.; M: Ind.; S: Nebensatz;

V: schwierig; mit präp. S.

176/37 gegeben worden wäre

N: Sg.; SF: bejahend; P: dritte; T: Pqp.; M: Kon.; S: Nebensatz;

V: leicht; ohne präp. S.

$177 / 23$ entladen worden

N: Pl.; SF: bejahend; P: dritte; T: Pqp.; M: Ind.; S: Nebensatz;

V: schwierig; ohne präp. S.

179/12 erfunden worden

N: Pl.; SF: bejahend; P: dritte; T: Pqp.; M: Ind.; S: Nebensatz;

V: schwierig; ohne präp. S.

183/37 wäre gesetzt worden

N: Sg.; SF: bejahend; P: dritte; T: Pqp.; M: Kon.; S: Hauptsatz;

V: schwierig; ohne präp. S.

188/5 wäre befördert worden

N: Sg.; SF: bejahend; P: nichtdritte; T: Pqp.; M: Kon.; S: Hauptsatz;

V: schwierig; ohne präp. S.

189/29 wären erkant worden

N: Pl.; SF: bejahend; P: dritte; T: Pqp.; M: Kon.; S: Hauptsatz;

V: schwierig; ohne präp. S. 
195/27 erschröckt worden

N: Sg.; SF: bejahend; P: dritte; T: Pqp.; M: Ind.; S: Nebensatz;

V: schwierig; ohne präp. S.

196/1 bezahlt worden

N: Pl.; SF: bejahend; P: dritte; T: Perf.; M: Ind.; S: Nebensatz;

V: schwierig; ohne präp. S.

$196 / 18$ verursacht worden

N: Sg.; SF: bejahend; P: dritte; T: Pqp.; M: Ind.; S: Nebensatz;

V: schwierig; mit präp. S.

202/10 muste offenbart worden seyn

infinite Form: S: Hauptsatz; V: schwierig; mit präp. S.

204/25 wäre commandirt worden

N: Sg.; SF: bejahend; P: nichtdritte; T: Pqp.; M: Kon.; S: Hauptsatz;

V: schwierig; ohne präp. S.

209/34 beschlossen worden

N: Sg.; SF: bejahend; P: dritte; T: Pqp.; M: Ind.; S: Nebensatz;

V: schwierig; ohne präp. S.

235/15 beredt worden

N: Pl.; SF: bejahend; P: dritte; T: Pqp.; M: Ind.; S: Nebensatz;

V: schwierig; mit präp. S.

235/21 gefangen worden

N: Sg.; SF: bejahend; P: dritte; T: Perf.; M: Ind.; S: Nebensatz;

V: leicht; ohne präp. S.

235/36 hinweg geschlagen worden wäre

N: Sg.; SF: bejahend; P: dritte; T: Pqp.; M: Kon.; S: Nebensatz;

V: schwierig; ohne präp. S.

versprochen worden wäre

N: Sg.; SF: bejahend; P: dritte; T: Pqp.; M: Kon.; S: Nebensatz;

V: schwierig; ohne präp. S.

250/3 getheilet worden

N: Pl.; SF: bejahend; P: dritte; T: Pqp.; M: Ind.; S: Nebensatz;

V: leicht; ohne präp. S. 
251/30 nicht her geholt worden wäre

N: Sg.; SF: nichtbejahend; P: nichtdritte; T: Pqp.; M: Kon.; S: Nebensatz;

V: schwierig; ohne präp. S.

$252 / 11$ gefangen worden

N: Sg.; SF: bejahend; P: nichtdritte; T: Pqp.; M: Ind.; S: Nebensatz;

V: leicht; ohne präp. S.

hingeführt worden

N: Pl.; SF: bejahend; P: nichtdritte; T: Pqp.; M: Ind.; S: Nebensatz;

V: schwierig; ohne präp. S.

252/17 ist eingehändigt worden

N: Sg.; SF: bejahend; P: dritte; T: Perf.; M: Ind.; S: Hauptsazt;

V: schwierig; mit präp. S.

255/31 wäre aussgezogen worden

N: Sg.; SF: bejahend; P: nichtdritte; T: Pqp.; M: Kon.; S: Nebensatz;

V: schwierig; ohne präp. S.

260/35 gefangen worden

N: Sg.; SF: bejahend; P: nichtdritte; T: Pqp.; M: Ind.; S: Nebensatz;

V: leicht; ohne präp. S.

$260 / 38$ bist vorgezogen worden

N: Sg.; SF: bejahend; P: nichtdritte; T: Perf.; M: Ind.; S: Nebensatz;

V: schwierig; mit präp. S.

$268 / 20$ ist erzogen worden

N: Sg.; SF: nichtbejahend; P: dritte; T: Perf.; M: Ind.; S: Hauptsatz;

V: schwierig; ohne präp. S.

ist unterrichtet worden

N: Sg.; SF: nichtbejahend; P: dritte; T: Perf.; M: Ind.; S: Hauptsatz;

V: schwierig; ohne präp. S.

268/36 gehalten worden

N: Sg.; SF: bejahend; P: dritte; T: Pqp.; M: Ind.; S: Nebensatz;

V: leicht; ohne präp. S.

270/24 begleitet worden

N: Pl.; SF: bejahend; P: dritte; T: Pqp.; M: Ind.; S: Nebensatz;

V: schwierig; mit präp. S. 
276/16 copulirt worden wärest

N: Sg.; SF: bejahend; P: nichtdritte; T: Pqp.; M: Kon.; S: Nebensatz;

V: schwierig; ohne präp. S.

277/37 angestellt worden wäre

N: Sg.; SF: bejahend; P: dritte; T: Pqp.; M: Kon.; S: Nebensatz;

V: schwierig; ohne präp. S.

279/8 geschlagen worden

N: Sg.; SF: bejahend; P: dritte; T: Pqp.; M: Ind.; S: Nebensatz;

V: leicht; ohne präp. S.

280/20 seyen petschirt worden

N: Pl.; SF: bejahend; P: dritte; T: Perf.; M: Kon.; S: Hauptsatz;

V: schwierig; ohne präp. S.

citirt worden

N: Sg.; SF: bejahend; P: dritte; T: Perf.; M: Kon.; S: Hauptsatz;

V: schwierig; ohne präp. $S$.

286/32 ist hinweg gefischt worden

N: Sg.; SF: bejahend; P: dritte; T: Perf.; M: Ind.; S: Hauptsatz;

V: schwierig; ohne präp. S.

293/18 geschickt worden ist

N: Sg.; SF: bejahend; P: dritte; T: Perf.; M: Ind.; S: Nebensatz;

V: leicht; ohne präp. $S$.

293/35 angestellt worden

N: Sg.; SF: bejahend; P: dritte; T: Perf.; M: Ind.; S: Nebensatz;

V: schwierig; ohne präp. S.

294/26 practicirt worden

N: Sg.; SF: bejahend; P: nichtdritte; T: Pqp.; M: Ind.; S: Nebensatz;

V: schwierig; ohne präp. S.

299/5 geschieden worden wären

N: Pl.; SF: bejahend; P: nichtdritte; T: Pqp.; M: Kon.; S: Nebensatz;

V: leicht; ohne präp. $S$.

299/25 wäre zugericht worden

N: Sg.; SF: bejahend; P: dritte; T: Pqp.; M: Kon.; S: Nebensatz;

V: schwierig; mit präp. S. 
301/34 ist nit beruffen worden

N: Sg.; SF: nichtbejahend; P: dritte; T: Perf.; M: Ind.; S: Hauptsatz;

V: schwierig; mit präp. S.

307/24 zugelassen worden

N: Sg.; SF: bejahend; P: dritte; T: Perf.; M: Ind.; S: Nebensatz;

V: schwierig; ohne präp. S.

319/29 erlöset worden

N: Sg.; SF: bejahend; P: nichtdritte; T: Perf.; M: Ind.; S: Nebensatz;

V: schwierig; ohne präp. S.

$321 / 16$ erzogen worden wäre

N: Sg.; SF: bejahend; P: nichtdritte; T: Pqp.; M: Kon.; S: Nebensatz;

V: schwierig; ohne präp. S.

323/21 geschickt worden

N: Sg.; SF: bejahend; P: dritte; T: Pqp.; N: Ind.; S: Nebensatz;

V: leicht; mit präp. S.

$324 / 22$ wäre erzogen worden

N: Sg.; SF: bejahend; P: nichtdritte; T: Pqp.; M: Kon.; S: Nebensatz;

V: schwierig; ohne präp. S.

338/9 sind nicht erraubt worden

N: Pl.; SF: nichtbejahend; P: dritte; T: Perf.; M: Ind.; S: Hauptsatz;

V: schwierig; ohne präp. S.

sind nicht zu wegen gebracht worden

N: Pl.; SF: nichtbejahend; P: dritte; T: Perf.; M: Ind.; S: Hauptsatz;

V: schwierig; ohne präp. S.

$338 / 13 z u$ wegen gebracht worden

N: Pl.; SF: bejahend; P: dritte; T: Perf.; M: Ind.; S: Nebensatz;

V: schwierig; mit präp. S.

$338 / 18$ seyen gerädert worden

N: Pl.; SF: bejahend; P: dritte; T: Perf.; M: Kon.; S: Nebensatz;

V: schwierig; ohne präp. S.

seyen gehenckt worden

N: Pl.; SF: bejahend; P: dritte; T: Perf.; M: Kon.; S: Nebensatz;

V: schwierig; ohne präp. S. 
seyen geköpfft worden

N: Pl.; SF: bejahend; P: dritte; T: Perf.; M: Kon.; S: Nebensatz;

V: schwierig; ohne präp. S.

340/1 ist erhört worden

N: Sg.; SF: nichtbejahend; P: dritte; T: Perf.; M: Ind.; S: Hauptsatz;

V: schwierig; ohne präp. S.

342/34 begangen worden

N: Pl.; SF: bejahend; P: dritte; T: Pqp.; M: Ind.; S: Nebensatz;

V: schwierig; ohne präp. S.

$345 / 14$ ist geboren worden

N: Sg.; SF: bejahend; P: dritte; T: Perf.; M: Ind.; S: Hauptsatz;

V: leicht; mit präp. S.

$360 / 8$ verspert worden

N: Pl.; SF: bejahend; P: dritte; T: Pqp.; M: Ind.; S: Nebensatz;

V: schwierig; ohne präp. S.

364/32 gefangen worden

N: Sg.; SF: bejahend; P: nichtdritte; T: Pqp.; M: Ind.; S: Nebensatz;

V: leicht; ohne präp. $S$.

untergestossen worden

N: Sg.; SF: bejahend; P: nichtdritte; T: Pqp.; M: Ind.; S: Nebensatz;

V: schwierig; ohne präp. S.

$365 / 16$ seyen überfallen worden

N: Pl.; SF: bejahend; P: nichtdritte; T: Perf.; M: Kon.; S: Hauptsatz;

V: schwierig; mit präp. S.

365/36 nicht geschmidet worden seye

N: Sg.; SF: nichtbejahend; P: dritte; T: Perf.; M: Kon.; S: Nebensatz

V: schwierig; mit präp. S.

nicht zugerichtet worden seye

N: Sg.; SF: nichtbejahend; P: dritte; T: Perf.; M: Kon.; S: Nebensatz;

V: schwierig; mit präp. S.

$367 / 12$ verbunden worden

N: Pl.; SF: bejahend; P: dritte; T: Pqp.; M: Ind.; S: Nebensatz;

V: schwierig; ohne präp. S. 
368/11 möchte verstossen worden seyn

infinite Form: S: Nebensatz; V: schwierig; ohne präp. S.

möchte gemacht worden seyn

infinite Form: S: Nebensatz; V: leicht; ohne präp. S.

möchte gesetzt worden seyn

infinite Form: S: Nebensatz; V: leicht; ohne präp. S.

374/24 erobert worden

N: Sg.; SF: bejahend; P: dritte; T: Pqp.; M: Ind.; S: Nebensatz;

V: schwierig; ohne präp. S.

384/19 gefangen worden

N: Sg.; SF: bejahend; P: dritte; T: Pqp.; M: Ind.; S: Nebensatz;

$\mathrm{V}$ : leicht; mit präp. S.

$386 / 20$ vergeben worden

N: Sg.; SF: bejahend; P: dritte; T: Pqp.; M: Ind.; S: Nebensatz;

V: schwierig; ohne präp. S.

$386 / 26$ vergeben worden wäre

N: Sg.; SF: bejahend; P: dritte; T: Pqp.; M: Kon.; S: Nebensatz;

V: schwierig; ohne präp. S.

386/30 bestochen worden

N: Sg.; SF: bejahend; P: dritte; T: Pqp.; M: Ind.; S: Nebensatz;

V: schwierig; mit präp. S.

394/29 vergeben worden

N: Sg.; SF: bejahend; P: dritte; T: Pqp.; M: Ind.; S: Nebensatz;

V: schwierig; ohne präp. S.

397/14 geboren worden

N: Sg.; SF: bejahend; P: dritte; T: Pqp.; M: Ind.; S: Nebensatz;

V: leicht; ohne präp. $S$.

403/22 geschnitten worden wäre

N: Sg.; SF: bejahend; P: dritte; T: Pqp.; M: Kon.; S: Nebensatz;

V: schwierig; ohne präp. S.

404/3 gestrafft worden wär

N: Sg.; SF: bejahend; P: nichtdritte; T: Pqp.; M: Kon.; S: Nebensatz;

V: leicht; ohne präp. S. 
N: Sg.; SF: bejahend; P: dritte; T: Pqp.; M: Ind.; S: Nebensatz;

V: leicht; ohne präp. S.

406/36 seye nicht gesehen worden

N: Sg.; SF: nichtbejahend; P: dritte; T: Perf.; M: Kon.; S: Hauptsatz;

V: leicht; ohne präp. S.

407/32 seye widerrathen worden

N: Sg.; SF: bejahend; P: dritte; T: Perf.; M: Kon.; S: Hauptsatz;

V: schwierig; mit präp. S.

seye hindertrieben worden

N: Sg.; SF: bejahend; P: dritte; T: Perf.; M: Kon.; S: Hauptsatz;

V: schwierig; mit präp. S.

408/15 nicht erfunden worden wäre

N: Sg.; SF: nichtbejahend; P: dritte; T: Pqp.; M: Kon.; S: Nebensatz;

V: schwierig; ohne präp. S.

$416 / 23$ seynd erschaffen worden

N: Pl.; SF: bejahend; P: dritte; T: Perf.; M: Ind.; S: Hauptsatz;

V: schwierig; ohne präp. S.

$416 / 29$ seynd erschaffen worden

N: Pl.; SF: bejahend; P: dritte; T: Perf.; M: Ind.; S: Hauptsatz;

V: schwierig; mit präp. S.

seynd begabt worden

N: Pl.; SF: bejahend; P: dritte; T: Perf.; M: Ind.; S: Hauptsatz;

V: schwierig; mit präp. S.

417/22 erschaffen worden

N: Pl.; SF: bejahend; P: nichtdritte; T: Perf.; M: Ind.; S: Nebensatz;

V: schwierig; mit präp. S.

geadelt worden

N: Pl.; SF: bejahend; P: nichtdritte; T: Pérf.; M: Ind.; S: Nebensatz;

V: schwierig; mit präp. S.

421/15 errathen worden wären

N: Pl.; SF: bejahend; P: dritte; T: Pqp.; M: Kon.; S: Nebensatz;

V: schwierig; mit präp. S. 
abgenommen worden wären

N: Pl; SF: bejahend; P: dritte; T: Pqp.; M: Kon.; S: Nebensatz;

V: schwierig; mit präp. S.

offenbart worden wären

N: Pl.; SF: bejahend; P: dritte; T: Pqp.; M: Kon.; S: Nebensatz;

V: schwierig; mit präp. $S$.

425/6 ist referirt worden

N: Sg.; SF: bejahend; P: dritte; T: Perf.; M: Ind.; S: Hauptsatz;

V: schwierig; ohne präp. S.

442/32 erbaut worden

N: Sg.; SF: bejahend; P: dritte; T: Pqp.; M: Ind.: S: Nebensatz;

V: schwierig; mit präp. $S$.

genennet worden

N: Sg.; SF: bejahend; P: dritte; T: Pqp.; M: Ind.; S: Nebensatz;

V: leicht; ohne präp. S.

$444 / 10$ gelegt worden

N: Sg.; SF: bejahend; P: dritte; T: Perf.; M: Ind.; S: Nebensatz;

V: leicht; ohne präp. S.

447/15 hindergangen worden

N: Sg.; SF: bejahend; P: nichtdritte; T: Pqp.; M: Ind.; S: Nebensatz;

V: schwierig; ohne präp. S.

453/2 solten überlassen worden seyn

infinite Form: S: Hauptsatz; V: schwierig; ohne präp. S.

455/28 war geschlossen worden

N: Sg.; SF: bejahend; P: dritte; T: Pqp; M: Ind.; S: Hauptsatz;

V: leicht; ohne präp. S.

479/18 nicht verfahren worden wäre

N: Sg.; SF: nichtbejahend; P: dritte; T: Pqp.; M: Kon.; S: Nebensatz;

V: schwierig; mit präp. S.

$482 / 12$ verstossen worden

N: Sg.; SF: bejahend; P: dritte; T: Perf.; M: Ind.; S: Nebensatz;

V: schwierig; ohne präp. S. 
$482 / 21$ nit abgedanckt worden

N: Sg.; SF: nichtbejahend; P: dritte; T: Perf.; M: Ind.; S: Nebensatz;

V: schwierig; ohne präp. S.

483/17 genennet worden

N: Sg.; SF: bejahend; P: dritte; T: Perf.; M: Ind.; S: Nebensatz;

V: leicht; ohne präp. S.

484/37 respectirt worden

N: Sg.; SF: bejahend; P: nichtdritte; T: Pqp.; M: Ind.; S: Nebensatz;

V: schwierig; ohne präp. S.

495/37 seye nit mitgeben worden

N: Sg.; SF: nichtbejahend; P: dritte; T: Perf.; M: Kon.; S: Hauptsatz;

V: schwierig; ohne präp. S.

496/14 verderbt worden

N: Sg.; SF: bejahend; P: dritte; T: Pqp.; M: Ind.; S: Nebensatz;

$\mathrm{V}$ : schwierig; ohne präp. $\mathrm{S}$.

verschwendet worden

N: Sg.; SF: bejahend; P: dritte; T: Pqp.; M: Ind.; S: Nebensatz;

V: schwierig; ohne präp. S.

501/4 vermacht worden

N: Sg.; SF: bejahend; P: dritte; T: Pqp.; M: Ind.; S: Nebensatz;

V: schwierig; mit präp. S.

502/35 müste geboren worden seyn

infinite Form: S: Nebensatz; V: leicht; ohne präp. S.

504/7 weggenommen worden wäre

N: Sg.; SF: bejahend; P: dritte; T: Pqp.; M: Kon; S: Nebensatz;

V: schwierig; mit präp. S.

504/14 ruinirt worden wäre

N: Sg.; SF: bejahend, P: dritte; T: Pqp.; M: Kon.; S: Nebensatz;

V: schwierig; mit präp. S.

gefangen worden wäre

N: Sg.; SF: bejahend; P: dritte; T: Pqp.; M: Kon.; S: Nebensatz;

V: leicht; mit präp. S. 
514/10 seynd gefunden worden

N: Pl.; SF: bejahend; P: dritte; T: Perf.; M: Kon.; S: Hauptsatz;

V: leicht; ohne präp. S.

seynd gezwungen worden

N: Pl.; SF: bejahend; P: dritte; T: Perf.; M: Kon.; S: Hauptsatz;

V: schwierig; ohne präp. S.

seynd genennet worden

N: Pl.; SF: bejahend; P: dritte; T: Perf.; M: Kon.; S: Hauptsatz;

V: leicht; ohne präp. S.

515/8 gezogen worden

N: Pl.; SF: bejahend; P: nichtdritte; T: Pqp.; M: Ind.; S: Nebensatz;

V: leicht; mit präp. S.

zusammen gekuppelt worden

N: Pl.; SF: bejahend; P: nichtdritte; T: Pqp.; M: Ind.; S: Nebensatz;

V: schwierig; mit präp. S.

516/32 gestampfft worden

N: Pl.; SF: bejahend; P: nichtdritte; T: Pqp.; M: Ind.; S: Nebensatz;

V: schwierig; ohne präp. S.

gestossen worden

N: Pl.; SF: bejahend; P: nichtdritte; T: Pqp.; M: Ind.; S: Nebensatz; V: leicht; ohne präp. S.

zerquetscht worden

N: Pl.; SF: bejahend; P: nichtdritte; T: Pqp.; M: Ind.; S: Nebensatz; V: schwierig; ohne präp. S.

geschwungen worden

N: Pl.; SF: bejahend; P: nichtdritte; T: Pqp.; M: Ind.; S: Nebensatz; V: leicht; ohne präp. S.

zerrieben worden

N: Pl.; SF: bejahend; P: nichtdritte; T: Pqp.; M: Ind.; S: Nebensatz; V: schwierig; ohne präp. S.

abgeplautet worden

N: Pl.; SF: bejahend; P: nichtdritte; T: Pqp.; M: Ind.; S: Nebensatz; V: schwierig; ohne präp. S. 
$522 / 3$ genommen worden ist

N: Sg.; SF: bejahend; P: nichtdritte; T: Perf.; M: Ind.; S: Nebensatz;

V: leicht; ohne präp. $S$.

523/15 aussgeben worden wäre

N: Sg.; SF: bejahend; P: dritte; T: Pqp.; M: Kon.; S: Nebensatz;

V: schwierig; mit präp. $S$.

$523 / 23$ gehalten worden

N: Sg.; SF: bejahend; P: dritte; T: Pqp.; M: Ind.; S: Nebensatz;

V: leicht; ohne präp. S.

$541 / 23$ zugeschickt worden wäre

N: Sg.; SF: bejahend; P: dritte; T: Pqp.; M: Kon.; S: Nebensatz;

V: schwierig; mit präp. S.

544/10 abgefertigt worden wäre

N: Sg.; SF: bejahend; P: dritte; T: Pqp.; M: Kon.; S: Nebensatz;

V: schwierig; ohne präp. S.

548/17 gefangen worden wäre

N: Sg.; SF: bejahend; P: dritte; T: Pqp.; M: Kon.; S: Nebensatz;

V: leicht; ohne präp. S.

549/19 genöttigt worden

N: Pl.; SF: bejahend; P: dritte; T: Pqp.; M: Ind.; S: Nebensatz;

V: schwierig; ohne präp. S.

$549 / 27$ gefangen worden

N: Pl.; SF: bejahend; P: dritte; T: Pqp.; M: Ind.; S: Nebensatz;

V: leicht; ohne präp. S.

verkaufft worden

N: Pl.; SF: bejahend; P: dritte; T: Pqp.; M: Ind.; S: Nebensatz;

V: schwierig; ohne präp. S.

549/35 eingehändigt worden

N: Sg.; SF: bejahend; P: dritte; T: Pqp.; M: Ind.; S: Nebensatz;

V: schwierig; ohne präp. S.

560/2 müste beraubt worden seyn

infinite Form: S: Nebensatz; V: schwierig; mit präp. S. 
$566 / 15$ hergebracht worden

N: Sg.; SF: bejahend; P: nichtdritte; T: Pqp.; M: Ind.; S: Nebensatz;

V: schwierig; ohne präp. S.

571/12 aussgesonnen worden

N: Sg.; SF: bejahend; P: dritte; T: Perf.; M: Ind.; S: Nebensatz;

V: schwierig; ohne präp. S.

beschlossen worden

N: Sg.; SF: bejahend; P: dritte; T: Perf.; M: Ind.; S: Nebensatz;

V: schwierig; ohne präp. S.

571/38 auffgerichtet worden

N: Sg.; SF: bejahend; P: dritte; T: Pqp.; M: Ind.; S: Nebensatz;

V: schwierig; mit präp. S.

$572 / 21$ gesetzt worden

N: Sg.; SF: bejahend; P: dritte; T: Pqp.; M: Ind.; S: Nebensatz;

V: leicht; ohne präp. S.

577/35 erschreckt worden

N: Sg.; SF: bejahend; P: dritte; T: Pqp.; M: Ind.; S: Nebensatz;

V: schwierig; mit präp. S.

gemüssigt worden

N: Sg.; SF: bejahend; P: dritte; T: Pqp.; M: Ind.; S: Nebensatz;

V: schwierig; mit präp. S.

577/37 molestirt worden

N: Sg.; SF: bejahend; P: dritte; T: Pqp.; M: Ind.; S: Nebensatz;

V: schwierig; mit präp. S.

$582 / 25$ verlohren worden

N: Sg.; SF: nichtbejahend; P: dritte; T: Pqp.; M: Ind.; S: Nebensatz;

V: leicht; ohne präp. S. 
Statistische Datenverarbeitung

worden-Passiv

Gesamtzahl der Belege: 217

davon finite Verbformen: 207

Infinitive: $10(4,5 \%)$

in bejahender Satzform: 178

in nichtbejahenden Satzformen: 29

$P_{(a 1)}=14 \%$

in Hauptsätzen: 50

in Nebensätzen: 167

$P_{(a 2)}=76 \%$

im Singular: 148

im Plural: 59

$P_{(\text {a3) }}=29 \%$

in der dritten Person: 158

in der nichtdritten Person: 49

$P_{(a 4)}=24 \%$

im Perfekt: 79

im Plusquamperfekt: 128

$P_{(\mathrm{a} 5)}=62 \%$

im Indikativ: 141

im Konjunktiv: 66

$P_{(\mathrm{a} 6)}=32 \%$

leichte Verben: 56

schwierige Verben: 161

$P_{(\mathrm{a} 7)}=75 \%$

ohne präp. S.: 159

mit präp. S.: 58

$P_{(\mathrm{a} 8)}=27 \%$

Passiv ohne worden und Aktiv

Gesamtzahl der Belege: 1155

davon Passivkonstruktionen ohne worden: 60 (5,2\%)

in bejahender Satzform: 1006

in nichtbejahenden Satzformen: 149

$P_{(a 1)}=13 \%$

in Hauptsätzen: 565

in Nebensätzen: 590

$P_{(\text {a2) }}=51 \%$ 
im Singular: 954

im Plural: 201

$P_{(\mathrm{a} 3)}=17 \%$

in der dritten Person: 702

in der nichtdritten Person: 453

$P_{(a 4)}=39 \%$

im Perfekt: 66

im Plusquamperfekt: 97

$P_{(\text {a5) }}=60 \%$

im Indikativ: 982

im Konjunktiv: 173

$P_{(a 6)}=15 \%$

leichte Verben: 776

schwierige Verben: 376

$P_{(a 7)}=33 \%$

ohne präp. S.: 53

mit präp. S.: 7

$P_{(a 8)}=12 \%$

Vergleich beider Samples

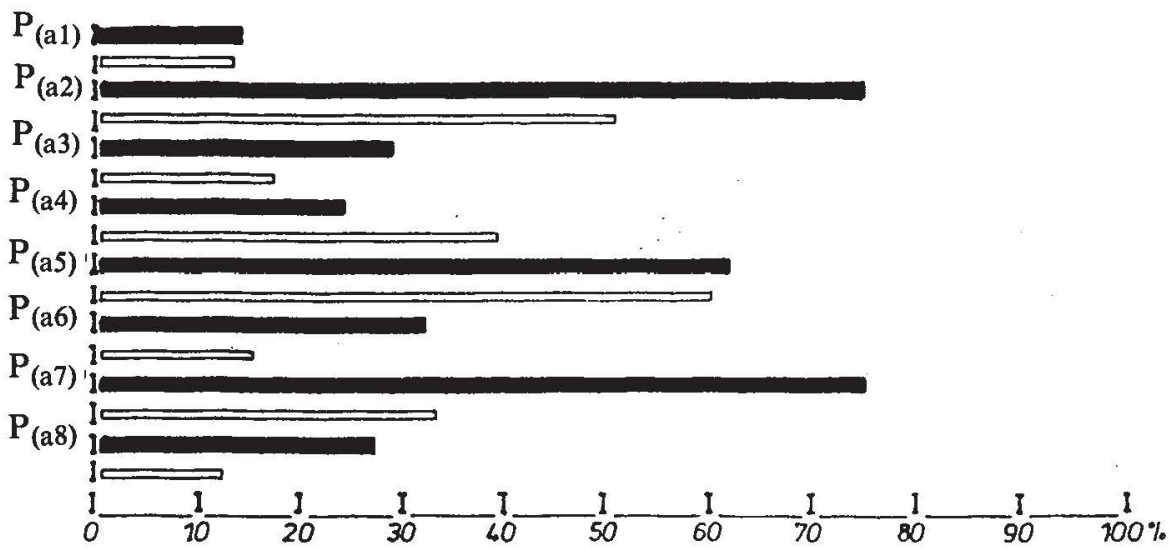

günstige Ereignisse

$\Longleftarrow$ ungünstige Ereignisse 
Das obige Histogramm zeigt eine deutlich grössere Tendenz des Vorkommens komplizierterer ("markierterer") grammatischer Verhältnisse beim worden-Passiv als beim Passiv ohne worden und beim Aktiv. Eine solche Konstellation zeigte sich innerhalb folgender grammatischer Parameter: Satzform (14\% gegenüber 13\%), Satzart ( $76 \%$ gegenüber $51 \%$ ), Numerus (29\% gegenüber $17 \%)$, Verbaltempus $(62 \%$ gegenüber $60 \%)$ und Modus (32\% gegenüber $15 \%)$. Die Komplexität der grammatischen Verhältnisse war beim worden-Passiv niedriger nur innerhalb des Parameters der Verbalperson (24\% gegenüber $39 \%$ ).

Beim worden-Passiv waren auch die Vollverben im Durchschnitt "schwieriger" als beim Passiv ohne worden und beim Aktiv (75\% gegenüber $33 \%$ ). Innerhalb aller Passiv-Periphrasen erscheint das worden-Passiv häufiger mit einem präpositionalen Agens (27\% gegenüber $12 \%$ ).

Vorkommenswahrscheinlichkeit "markierterer" Werte einzelner grammatischer Kategorien:

$\begin{array}{llll}\text { 1. nichtbejahende Sätze: } & \mathrm{P}_{(\mathrm{a} 1)}: 14 \% 0^{3} & 13 \% 0^{4} & \mathrm{Id}^{5}=0,43 \\ \text { 2. Nebensatz: } & \mathrm{P}_{(\mathrm{a} 2)}: 76 \% & 51 \% & \text { Id }=7,01 \\ \text { 3. Plural: } & \mathrm{P}_{(\mathrm{a})}: 29 \% & 17 \% & \text { Id }=3,64 \\ \text { 4. nichtdritte Person: } & \mathrm{P}_{(\mathrm{a} 4)}: 24 \% & 39 \% & \text { Id }=4,44 \\ \text { 5. Plusquamperfekt: } & \mathrm{P}_{(\mathrm{a}))}: 62 \% & 60 \% & \text { Id }=0,55 \\ \text { 6. Konjunktiv: } & \mathrm{P}_{(\mathrm{a})}: 32 \% & 15 \% & \text { Id }=5,29 \\ \text { 7. "schwierige" Vollverben: } & \mathrm{P}_{(\mathrm{a})}: 75 \% & 33 \% & \text { Id }=11,47 \\ \text { 8. mit präp. Agens: } & \mathrm{P}_{(\mathrm{a} 8)}: 27 \% & 12 \% 0^{6} & \text { Id }=2,62\end{array}$

Vergleich der durch die Korpusanalyse gewonnenen Ergebnisse mit den Vorhersagen

Mit der Einführung zusammengesetzter Tempora in das deutsche Verbalsystem zeigt sich das Bedürfnis nach Ausdehnung des Passiv-Paradigmas in zusammengesetzte (kompliziertere) Verbaltempora als Analogiebildung zum Aktiv. Die Folge des so entstandenen Systemdrucks ist die Einführung des worden-Passivs. Diese periphrastische Konstruktion verstärkt ihrerseits das Passiv-Paradigma mit einem neuen Element. Sie erscheint als verstärke Konstruktion mit dem höchsten Markiertheitsgrad innerhalb des Passivs, und als solche verdrängt sie allmählich die weniger markierte / geschwächtere Variante zur Bezeichnung der Perfektivität, d.h. der Typ ist erschossen / bewohnt; dieses weicht in eine "leichtere" grammatische Umgebung - ins Präsens. Durch die Grammatikalisierung des worden-Passivs wird die paradigmatische Instabilität abgeschafft.

\footnotetext{
worden-Passiv

Passiv ohne worden und Aktiv

Id = Index der Verschiedenheit (nur bei Id $\geqslant 2$ ist der Unterschied statistisch signifikant)

Passiv ohne worden
} 
In der mittelhochdeutschen Periode setzt sich das worden-Passiv als Verstärkung des Passiv-Paradigmas zunächst in relativ komplizierten grammatischen Verhältnissen und im komplizierten Kontext durch:

Beobachtet wurde auch die Beschaffenheit des sprachlichen Materials, welches im syntaktischen Wandel auftritt. Dabei konnte durch die Datenauswertung die Grundhypothese bestätigt werden, wonach alle jene syntaktischen Verstärkungen in der Sprache, die ein Verbum als Zentralwort aufweisen, zunächst bei "schwierigeren" Verben auftreten. Die Komplexität der grammatischen Verhältnisse und des Kontextes beim worden-Passiv wurde zwar anhand eines Sprachmaterials aus dem 17. Jh. überprüft, d.h. aus einer Zeit, wo die Grammatikalisierung wohl schon abgeschlossen war. ${ }^{7}$ Es zeigte sich jedoch noch immer eine grössere Häufigkeit zugunsten "schwierigerer" Verben: bei der beobachteten verstärkten Konstruktion konnten ganze $75 \%$ (Id $=11,47 \%$ ) festgestellt werden.

Die Resultate der Korpusanalyse bestätigten auch die Grundhypothese, welche besagt, dass sich verstärkte Konstruktionen zunächst im komplizierten Kontext durchsetzen. Diesbezüglich wurden acht grammatische Parameter untersucht, wovon fünf $(62,5 \%)$ in der verstärkten Konstruktion signifikant häufiger auftraten als bei Konstruktionen, welche im Vergleich mit dem worden-Passiv als "nichtverstärkte" aufgefasst wurden (Aktiv, Passiv ohne worden). Bei zwei grammatischen Parametern (nichtbejahender Satzform und im Plusquamperfekt) lag die Häufigkeit der verstärkten Konstruktion über der Häufigkeit der "nichtverstärkten" Konstruktionen, jedoch war hier der festgestellte Unterschied statistisch nicht signifikant. Bei der grammatischen Kategorie der Verbalperson hingegen war die Häufigkeit der verstärkten Konstruktion in der komplizierteren Variante (nichtdritte Person) signifikant niedriger.

Die Tatsache, dass die Vorhersage im Fall der Satzform und des Verbaltempus nur teilweise bestätigt und im Fall der Verbalperson widerlegt wurde, legen wir als Folgeerscheinung der Charakteristika des beobachteten Sprachmaterials aus. Dieses ist nämlich relativ weit entfernt vom natürlichen Gesprächsablauf, auf den sich unsere Vorhersagen eigentlich beziehen. Eine zweite (oder zusätzliche) Möglichkeit der Interpretation weist in eine Richtung, der zufolge man die Kompliziertheitsgrade nicht nur innerhalb einzelner grammatischer Kategorien unterscheiden, sondern sie auch in ihrem gegenseitigen Verhältnis auseinanderhalten soll, d.h. der Kompliziertheitsgrad einzelner grammatischer Kategorien vermag sich nicht nur horizontal, sondern auch vertikal zu bewegen. (In diesem Fall wäre etwa der Parameter der Verbalperson weniger kompliziert als beispielsweise der Parameter der Satzart.) Diese Hypothese bedarf jedoch einer Überprüfung durch verschiedene Korpusanalysen in mehreren Sprachen.

7 Die ältesten Belege konnten nicht beobachtet werden, da sie selten auftreten und man daher keine zuverlässigen statistischen Untersuchungen vornehmen könnte. 
Die Vorhersage wurde auch im Fall der Infinitiv-Konstruktionen bestätigt, welche als "leichterer" Kontext aufgefasst wurden. Im Infinitiv trat das worden-Passiv nur in $4,6 \%$ auf, dagegen Passiv ohne worden in $8,3 \%(\mathrm{Id}=1,10$ ).

Im untersuchten Korpus befindet sich die Entwicklung der verstärkten Konstruktion, d.h. des worden-Passivs, in einer Phase, in welcher ihr Auftreten noch nicht alle theoretisch möglichen Stellen erreicht hatte. Dabei denken wir in erster Linie an "leichtere" Wörter bzw. Formen.

Generelle Wertung der Arbeitshypothese und Ausblick

Aus dem bisher Dargelegten ist zu ersehen, dass die Theorie vom Sprachwandel, welcher sich in Form von verstärkten und geschwächten Konstruktionen vollzieht und durchsetzt, auch in der Syntax ihre Gültigkeit findet. Um diese Theorie zu untermauern, wären weitere Forschungsarbeiten erforderlich, die andere syntaktische Veränderungen erfassen würden, beispielsweise auch die Endstufe ihrer Entwicklung, bis hin zum möglichen Ausklingen einzelner syntaktischer Erscheinungen.

Die Erörterung des syntaktischen Wandels ist mit der gegenwärtigen Untersuchung natürlich noch nicht erschöpft. Es wird nur eine Teilantwort auf die Frage angeboten, auf welche Art und Weise sich der syntaktische Wandel auf natürlichem Wege vollzieht (konkret: SYNTAKTISCHE VERSTÄRKUNGEN), die Ursachen und Gründe für sein Entstehen und Durchsetzen bleiben jedoch unberücktsichtigt.

\section{ZUSAMMENFASSUNG}

Die Sprache veränert sich an jenen Stellen, an denen ihre GrundfunktionInformationsaustausch, gestört wird. Wenn einer sprachlichen Veränderung das Bestreben nach grösserer Deutlichkeit zugrunde liegt, sprechen wir (auf die Syntax bezogen) vom Durchsetzen verstärkter Konstruktionen; wenn jedoch sprachliche Veränderungen ökonomischeres Sprechen / Schreiben zur Folge haben, sprechen wir (auf die Syntax bezogen) vom Durchsetzen geschwächter Konstruktionen. (Periphrasen wären etwa ein typisches Beispiel verstärkter Konstruktionen.) Jede der beiden Arten des Sprachwandels setzt sich nach ihr eigenen / verschiedenen Gesetzmässigkeiten durch. Generell ist anzunehmen, dass sich verstärkte Konstruktionen am Anfang vorzugsweise unter relativ komplizierten grammatischen Verhältnissen durchsetzen (und sich später möglicherweise auch unter weniger komplizierten grammatischen Verhältnissen verbreiten). Geschwächte Konstruktionen behaupten sich dagegen am Anfang vorzugsweise unter relativ einfachen grammatischen Verhältnissen (und verbreiten sich später möglicherweise auch unter weniger einfachen grammatischen Verhältnissen). Diese Betrachtungsweise setzte sich in der Phonologie und Morphologie durch, unterblieb aber bislang in der Syntax. 
Der Hauptzweck der gegenwärtigen Untersuchung war die Erforschung der Verhaltensweisen einer syntaktischen Veränderung / verstärkten Konstruktion in der Anfangsstufe ihrer Entwicklung. Dabei wurde festzustellen versucht, ob der Tatbestand den das Durchsetzen von verstärkten Konstruktionen in einer Sprache regelnden Gesetzmässigkeiten entspricht. Untersucht wurde das worden-Passiv, welches als .Periphrase der Definition der verstärkten Konstruktionen entspricht. Als Korpus wurde Grimmelshausens Simplicissimus gewählt (17. Jahrhundert).

Das deutsche worden-Pasiv ist im untersuchten Sprachmaterial, im Vergleich zum Aktiv und Passiv ohne worden, signifikant häufiger im Nebensatz als im Hauptsatz aufgetreten, weiters im Plural häufiger als im Singular, im Konjunktiv häufiger als im Indikativ, im Kontext mit "schwierigeren" Verben häufiger als im Kontext mit "leichteren" Verben und mit präpositionalem Agens häufiger als ohne dasselbe. Die Häufigkeit des Vorkommens "schwieriger" grammatischer Kategorien zugunsten der verstärkten Konstruktion war statistisch nicht signifikant bei der nichtbejahenden Satzform und beim Plusquamperfekt. Die Häufigkeit des Vorkommens neigte sich zugunsten der weniger verstärkten Konstruktionen lediglich bei der nichtdritten Person (in diesem Fall wurde die entsprechende Vorhersage nicht bestätigt).

Das bisher Darglegte zeigt, dass das worden-Passiv mit einer 87,5\%-igen Häufigkeit unter komplizierten grammatischen Verhältnissen auftrat, dies aber entspricht den für verstärkte Konstruktionen geltenden Gesetzmässigkeiten.

Somit scheinen die Resultate der Korpusanalyse die Richtigkeit der Annahme zu bestätigen, dass die Theorie über sprachliche Veränderungen, die sich als verstärkte oder geschwächte Formen / Konstruktionen durchsetzen, auch auf Syntax ausgedehnt werden kann. Die Gültigkeit dieser Theorie bedarf jedoch noch einer Bestätigung mittels Untersuchungen weiterer syntaktischer Veränderungen (verstärkter oder geschwächter Konstruktionen), nach Möglichkeit unter Einbeziehung verschiedener Sprachen.

\section{LITERATUR}

Behaghel O., Deutsche Syntax, Carl Winter, Heidelberg 1924-1923.

Braune W., Althochdeutsche Grammatik, dritte und vierte Auflage, Max Niemeyer, Halle a./S. 1911.

Brinker K., Das Passiv im heutigen Deutsch, Hueber und Schwann, München und Düsseldorf 1971.

Dal I., Kurze deutsche Syntax auf historischer Grundlage, Max Niemeyer, Tübingen 1966.

Eggers H., Mittelhochdeutsche Grammatik, VEB Bibliographisches Institut, Leipzig 1970. 
Eichinger L.M., Zum Passiv im althochdeutschen Isidor, in Akten des Kolloquiums über das Passiv im Deutschen: Das Passiv im Deutschen, Nizza 1986, herausgegeben vom Centre de Recherche en Linguistique (Nice), Max Niemeyer, Tübingen 1987.

Grimmelshausen H. J. C., Der Abentheurliche Simplicissimus Teutsch und Continuatio des abentheurlichen Simplicissimi, 2., durchgesehene und erweiterte Auflage, herausgegeben von R. Tarot, Max Niemeyer, Tübingen 1984.

Luther M., Das Neue Testament - unsers Herrn und Heilandes Jesu Christi, Britische und Ausländische Bibelgesellschaft, Berlin und Köln 1892.

OEberg A.B., Über die hochdeutsche Passivumschreibung mit sein und werden. Historische Darstellung. Akademische Abhandlung, Berlingska Boktryckeriet, Lund 1907.

Rupp H., Zum Passiv im Althochdeutschen, in: Beiträge zur Geschichte der deutschen Sprache und Literatur, 78, Halle, 1956.

Schönthal G., Das Passiv in der deutschen Standardsprache, Hueber, München 1976.

Tarot R., siehe Grimmelshausen.

Valentin P., Zur Geschichte des deutschen Passivs, in Akten des Kolloquiums über das Passiv im Deutschen: Das Passiv im Deutschen, Nizza 1986, herausgegeben vom Centre de Recherche en Linguistique (Nice), Max Niemeyer, Tübingen 1987.

\section{Povzetek}

\section{TRPNIK Z WORDEN KOT SKLADENJSKA OKREPITEV V NEMŠČINI}

Osnovni namen naloge je bil opazovati skladenjske spremembe (sistemsko okrepitev) in njeno vedenje na začetni stopnji razvoja (vendar nismo opazovali samega nastanka skladenjske spremembe). Pri tem smo ugotavljali, ali okoliščine ustrezajo zakonitostim, po katerih naj bi se $v$ jeziku uveljavljale okrepitve. Skladenjsko okrepitev smo opazovali pri nemškem trpniku z worden, ki kot opisna zgradba ustreza definiciji okrepitev. Analizirali smo "Simplicissimusa", avtorja Hansa Jacoba von Grimmelshausena (17. stoletje).

Nemški trpnik $z$ worden se je v obdelanem jezikovnem gradivu, za razliko od trpnika brez worden in tvornika, signifikantno pogosteje pojavljal $v$ odvisnem stavku kot $v$ glavnem, $v$ neednini kot $v$ ednini, $v$ konjunktivu kot v indikativu, s "težkimi" glagoli kot $\mathrm{z}$ "lahkimi" in s predložnim osebkom kot brez le-tega. Pogostnost krepkejših oblik ni bila statistično signifikantna v netrdilni obliki stavka in predpretekliku, v manj okrepljene zgradbe pa se je pogostnost prevesila le $v$ netretji osebi (tod ustrezna delovna napoved ni bila potrjena).

Analiza kaže, da se je trpnik $z$ worden $\vee 87,5 \%$ pojavljal v zapletenem okolju in s "težko" glavno glagolsko obliko pogosteje kot v manj zapletenem, to pa je v skladu z zakonitostmi, ki naj bi urejevale uveljavljanje krepkejših zgradb.

Rezultati, dobljeni pri obdelavi izbranega jezikovnega gradiva, so pokazali, da lahko teorijo o jezikovnih spremembah, ki se pojavljajo in uveljavljajo kot sistemske okrepitve in sistemske ošibitve, razširimo tudi na skladnjo. Veljavnost teorije pa bi bilo treba potrditi še z raziskavami nadaljnjih skladenjskih sprememb (krepkejših in کibkejših zgradb), po možnosti v različnih jezikih. 